\title{
Technique Selection 'the Coaches Challenge' Influencing Injury Risk During the First Contact Hand of the Round off Skill in Female Gymnastics
}

\author{
by \\ Roman Farana ${ }^{1}$, Daniel Jandacka ${ }^{1}$, Jaroslav Uchytil ${ }^{1}$, David Zahradnik ${ }^{1}$, \\ Gareth Irwin ${ }^{1,2}$
}

\begin{abstract}
The importance of technique selection on elbow injury risk has been identified for the key round off skill in female gymnastics, with a focus on the second contact limb. The aim of this study was to shift the focus to the first contact limb and investigate the biomechanical injury risk during parallel and T-shape round-off (RO) techniques. Seven international-level female gymnasts performed 10 trials of the $R O$ to back-handspring with parallel and T-shape hand positions. Synchronized kinematic (3D motion analysis system; $247 \mathrm{~Hz}$ ) and kinetic (two force plates; $1235 \mathrm{~Hz}$ ) data were collected for each trial. The t-test with effect size statistics determined differences between the two techniques. No significant differences were found for vertical, anterior posterior and resultant ground reaction force, elbow joint kinematics and kinetics. Specifically, the results highlighted that change in technique in RO skills did not influence first contact limb elbow joint mechanics and therefore, injury risk. The findings of the present study suggest the injury potential of this skill is focused on the second limb during the parallel technique of this fundamental gymnastic skill.
\end{abstract}

Key words: gymnastics, fundamental skill, upper extremity, prevention.

\section{Introduction}

A unique aspect of gymnastics is the need for the upper extremities to support gymnast's full body weight (DiFiori et al., 2006). During skills and routines, gymnasts land repetitively on the hands whilst tumbling on the floor and performing vaulting (Daly et al., 1999). The consequence of upper limbs being weight-bearing causes high impact loads to be distributed through the wrist and elbow (Webb and Rettig, 2008); these repetitive loads can lead to both acute and chronic injuries (Davidson et al., 2005). There is previous evidence that a major career ending injury site in female gymnastics is the elbow joint complex (Chan et al., 1991; Jackson et al., 1989), which is susceptible to micro traumatic lesions and typically stems from the abduction load (Hume et al., 2006; Koh et al., 1992). Furthermore,
Magra et al. (2007) demonstrated that the abduction position and corresponding internal adduction moment of the elbow produced abduction loading and probably contributed to some of the overuse injury patterns such as valgus extension overloading. These repetitive loads cause lesions to the elbow, including medial collateral ligament strains, medial epicondyle traction injuries and osteochondritis dissecans of the capitellum (Frostick et al., 1999; Koh et al., 1992). Chronic injuries resulting from skills that are well learned, basic or moderately difficult have been shown to be most common, and these occur with highest frequency on the floor exercise (Lindner and Caine, 1990). The major challenge for coaches and athletes is the selection of technique, considering that the same skill can be

1 - Department of Human Movement Studies, Human Motion Diagnostic Center, University of Ostrava, Czech Republic.

2 - Sport Biomechanics Research Group, Cardiff School of Sport, Cardiff Metropolitan University, Cardiff, UK. 
performed with a number of different techniques. Technique selection may have an impact on injury and the evolution of the skill and is an important area for research (Cossens, 2012; Farana et al., 2014, 2016).

The round-off (RO) (Fédération Internationale de Gymnastique) is a fundamental gymnastics skill and a key movement in the development of elite female gymnasts, owing to its association with learning more complex skills. Two common techniques are used to perform RO, the parallel hand position and the T-shape hand position (Figure 1).

Injury risk and skill technique selection have been examined in technical coaching articles (Sands and McNeal, 2006) and empirical biomechanics research (Farana et al., 2014, 2016), in which the risk associated with the choice of hand placement during a back handspring and the RO was demonstrated, respectively. Sands and McNeal (2006) suggested that by turning the hands inward during a back handspring, the female gymnast reduced both the risk of injuring the elbow and the risk of damage to the wrist. Farana et al. (2014) and Farana et al. (2015) showed that different hand positions during the RO among female gymnasts significantly influenced elbow loading and biological variability on the second contact limb. More specifically, the T-shape position of the hands reduced peak vertical, anterior-posterior, and resultant ground reaction forces (GRFs), decreased loading rates and internal adduction moment indicating a safer technique for the RO. However, a limitation of this previous research is that only the second contact limb during the RO was investigated and there is, to date, no information about first contact limb mechanics. Therefore, there is a need to examine the first contact limb mechanics and the role it plays. For example, during the T-shape technique when, as previously reported (Farana et al., 2014), there is a significant decrease in the peak GRFs and elbow joint abduction loading, it remains unknown whether the first limb acts as an associated compensatory mechanism. Hence, this study focused on examination of the first contact limb and the effect of different hand positions on the injury risk factors.

In a study that examined ground reaction forces transmitted to the upper extremities,
Panzer et al. (1987) found that during the Tsukahara vault, elbow joint reaction forces ranged from 1.7 to $2.2 \mathrm{BW}$. Moreover, Seeley and Bressel (2005) observed a bi-modal force trace depicting vertical reaction forces (VGRF) for the first hand and then the support phase during the round-off of a Yurchenko vault. However, these authors did not use separate force plates for each hand and as such, could not fully comment on the underlying mechanics and injury risk. To our knowledge, there has been no detailed investigation of the first contact limb mechanics during the RO skills which may have implications for injury risk. In general, there is a lack of research focused on the interaction between impacting upper limbs in sports like gymnastics. The need for this research is supported theoretically to develop a better understanding of the stochastic nature of injury.

Therefore, the aim of this study was to investigate injury risk factors including impact forces, elbow joint kinetics and kinematics in the first contact limb for both parallel and T-shape RO techniques. This research hypothesized that variation in the hand position of the second contact limb would affect mechanics of the first contact limb elbow joint which may be related to injury. The data presented in this paper were obtained from the same subjects as in Farana et al.'s (2014) study, in which changes in impact loading as well as elbow kinematics and kinetics for the second contact limb were investigated. Such a sample choice, in our opinion, will facilitate a meaningful comparison. The current research provides original insight into technique selection and the potential interaction of support limbs during fundamental skills in female gymnastics.

\section{Material and Methods}

\section{Participants and Protocol}

Seven international level female gymnasts participated in this study. The gymnasts were members of the junior and senior national gymnastics team of the Czech Republic with average training and competition experience of 14 \pm 2 years. Their mean $( \pm \mathrm{SD})$ body height was $162.9 \pm 3.9 \mathrm{~cm}$; body mass $56.7 \pm 5.2 \mathrm{~kg}$ and age $20.7 \pm 1.6$ years. All gymnasts were injury free at the time of testing. More details about preferred technique of each gymnast were previously 
described by Farana et al. (2014). Informed consent from the participants was obtained before the commencement of the study which was performed in accordance with the guidelines of the Ethics and Research Committee of the Human Motion Diagnostic Centre. The research was conducted in the Biomechanical Laboratory. The gymnasts completed their self-selected warm up and completed a number of practice RO trials using both techniques. A thin gymnastic floor cover mat (dimension $20 \mathrm{~mm}$, Baenfer, Germany) was used that was taped down onto each force plate to replicate the feel of a typical gymnastics' floor. Additionally, landing mats were used to provide safety for the gymnasts' landing. After the warm up and practice, all gymnasts performed 10 trials of RO with a parallel hand position from a hurdle step to a back handspring, and 10 trials of $\mathrm{RO}$ with a T-shape hand position from a hurdle step to a back handspring in random order and separated by a one-minute rest period.

\section{Measures}

Two force plates (Kistler, Switzerland) embedded into the floor determined GRF at a sampling rate of $1235 \mathrm{~Hz}$. Depth of the transducer was set as the sum of the manufacturer depth for the specific force plate and the depth of the mat (Farana et al., 2014). The force plates were synchronized with a motion-capture system (Qualisys Oqus, Sweden) consisting of eight infrared cameras collecting kinematic data at a sampling rate of $247 \mathrm{~Hz}$. The global coordinate system was set with the $z$-axis as vertical, $y$-axis as anterior-posterior and $\mathrm{x}$-axis as medio-lateral. Retroreflective markers and two clusters containing three markers each (19 mm diameter) were attached to the gymnasts' upper limbs and trunk (C-motion, Rockville, MD, USA), they were also placed bilaterally on the upper arm and forearm (Figure 2). Markers and clusters were bilaterally placed on each participant at the anatomical locations previously described by Farana et al. (2014). Two photocell timing gates were used to control hurdle step horizontal velocity which was standardized at range of 3.3 to $3.7 \mathrm{~m} / \mathrm{s}$ (Farana et al., 2013).

Raw coordinate data were processed using Visual 3D software (version 4; C-motion, Rockville, MD, USA). All upper extremity segments were modelled as a frusta of right circular cones and the trunk as an elliptical column. The local coordinate systems were defined using a static calibration trial in the handstand position. All analyses focused on the contact phase of the first contact hand during the RO. Kinematic variables included sagittal (+ flexion, - extension), frontal (+ adduction, abduction) and transverse (+ internal rotation, external rotation) elbow angles and these were calculated using XYZ (mediolateralanteroposterior-longitudinal) order of rotation. Kinetic variables included peak vertical GRF (VGRF), anterior-posterior GRF (APGRF), resultant GRF (RGRF), and loading rates of these forces. In addition, net three-dimensional internal elbow joint moments in the sagittal (+ flexion, extension), frontal (+ adduction, - abduction), and transversal $(+$ internal rotation, - external rotation) planes were quantified by the NewtonEuler inverse dynamics technique, using the segmental inertial characteristics, hand, forearm and upper arm markers positions, and GRFs during first hand contact time (Selbie et al., 2014). Net internal elbow moments were expressed in the local coordinate system of the upper arm. The coordinate and force plate data were low-pass filtered using a fourth-order Butterworth filter with a $12 \mathrm{~Hz}$ and $50 \mathrm{~Hz}$ cut off frequency, respectively. The GRF and moment data were normalised to body mass.

\section{Statistical Analysis}

Statistical tests were used to examine the effects caused by the independent variables "hand position" (a parallel hand position versus a Tshape hand position) on the dependent variables (i.e., impact forces, elbow joint angles and moments of force) of the first contact hand. Mean values of the 10 trials for each gymnast in each technique were calculated for all measured variables and used in statistical analysis. The Shapiro-Wilk test confirmed the normality assumption for the data and a paired t-test was performed. The level of significance was set at $p<$ 0.05. Effect sizes (ES) were calculated using Cohen's $d$ and presented as $<0.2$ trivial; $0.21-0.5$ small; $0.51-0.8$ medium and $>0.8$ large (Cohen, 1988). To provide further information regarding differences between parallel and T-shape hand positions, the $95 \%$ confidence interval (CI) of the mean difference was derived in order to minimise the occurrence of a type I error. Statistical tests 
were processed using IBM SPSS Statistics 20 Software (IBM SPSS Inc., Chicago, IL, USA).

\section{Results}

Descriptive statistics with means, standard deviations and statistical results for the two techniques and the first contact limb are presented in Table 1. No significant differences were found for peak VGRF, APGRF, RGRF (Figure 3A) and VRGF loading rates (Figure 3B). As shown in Figure $3 \mathrm{~B}$, a significant difference, although small effect sizes were found for peak APGRF ( $p=0.033$, $E S=0.40)$ and RGRF loading rates $(p=0.025, E S=$ 0.22 ) for the parallel hand position. Furthermore, no significant differences were observed for peak elbow flexion, abduction and internal rotation angles (Figure 3C), and corresponding internal extension, adduction and external rotation moments (Figure 3D).
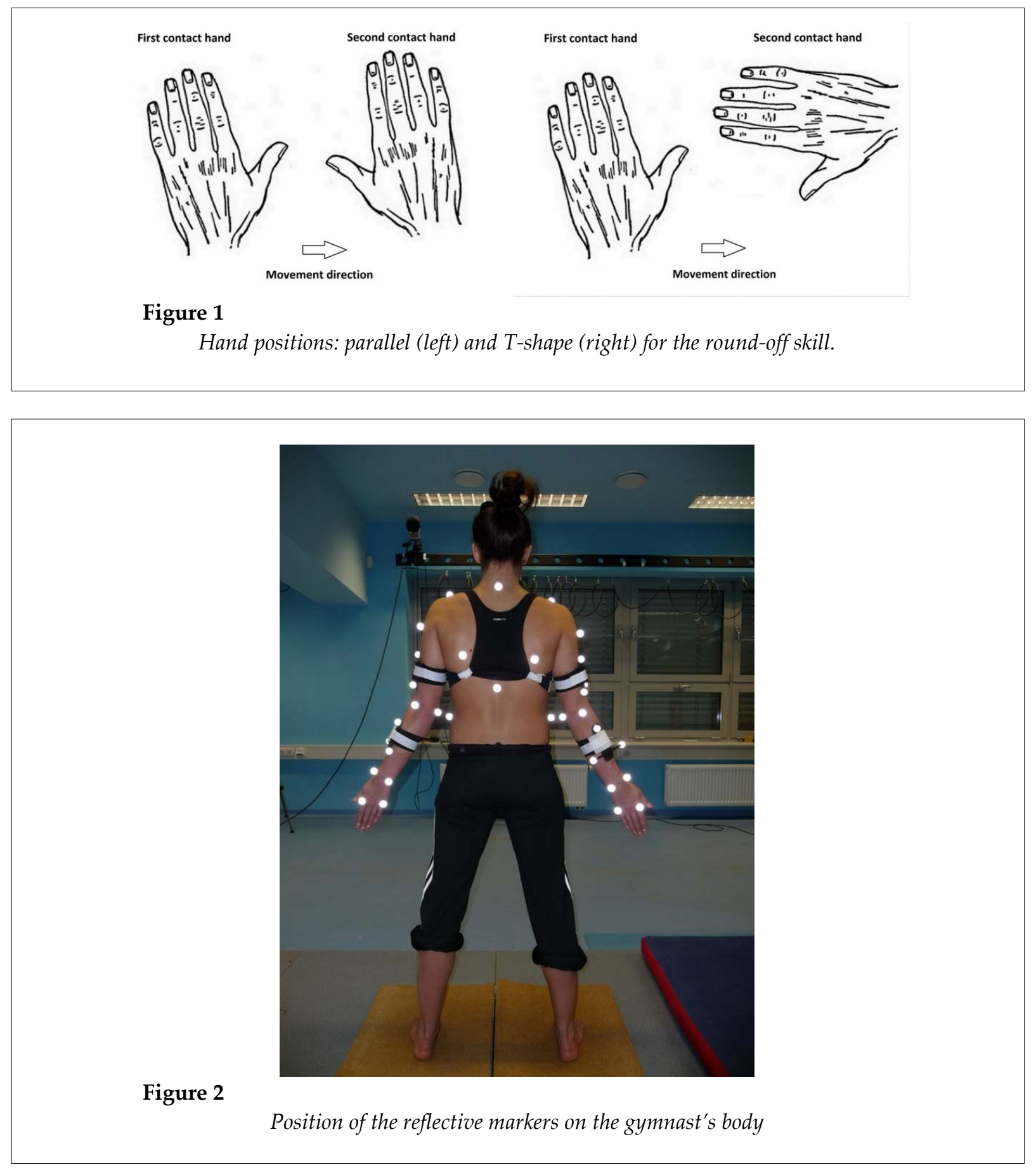


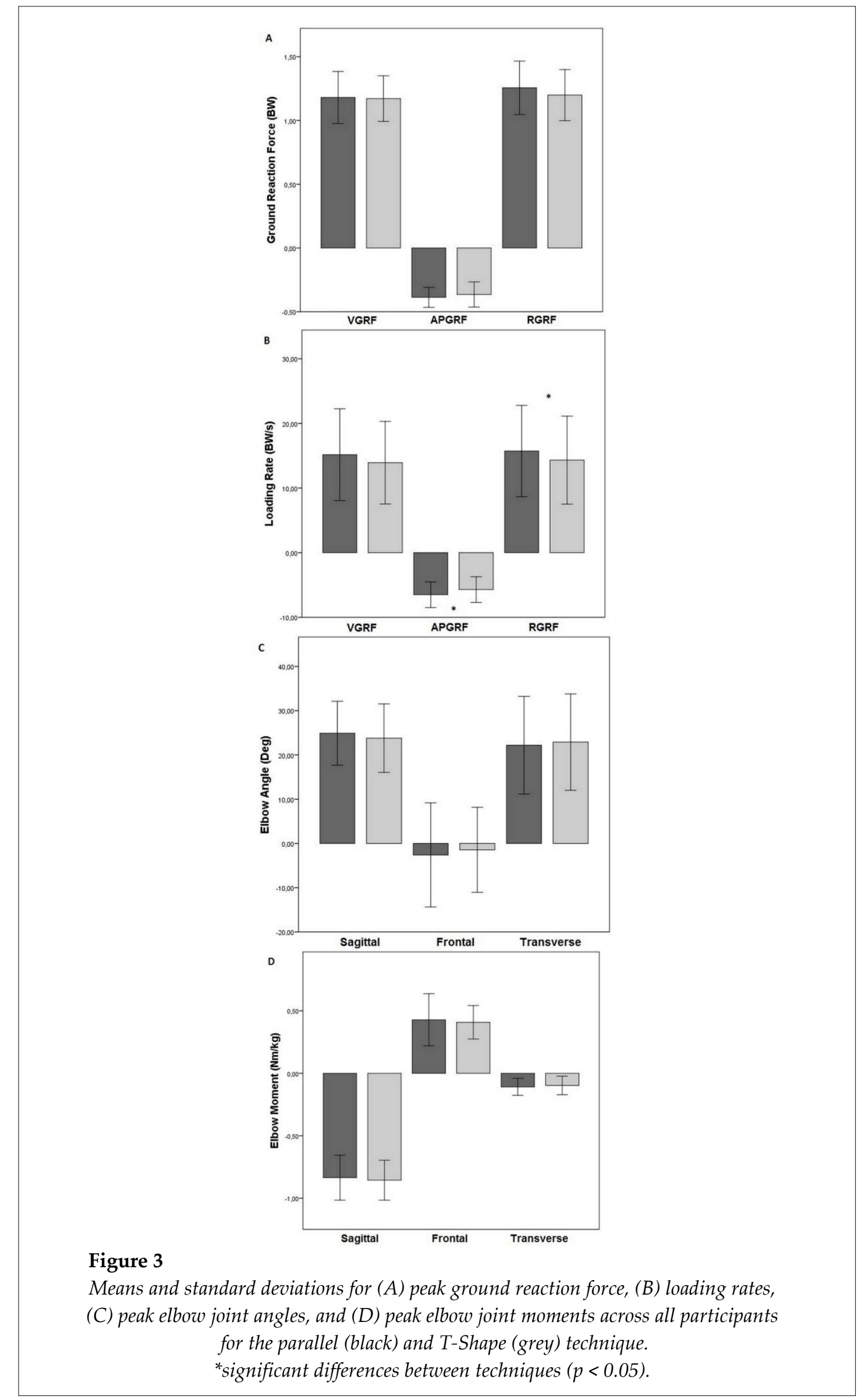




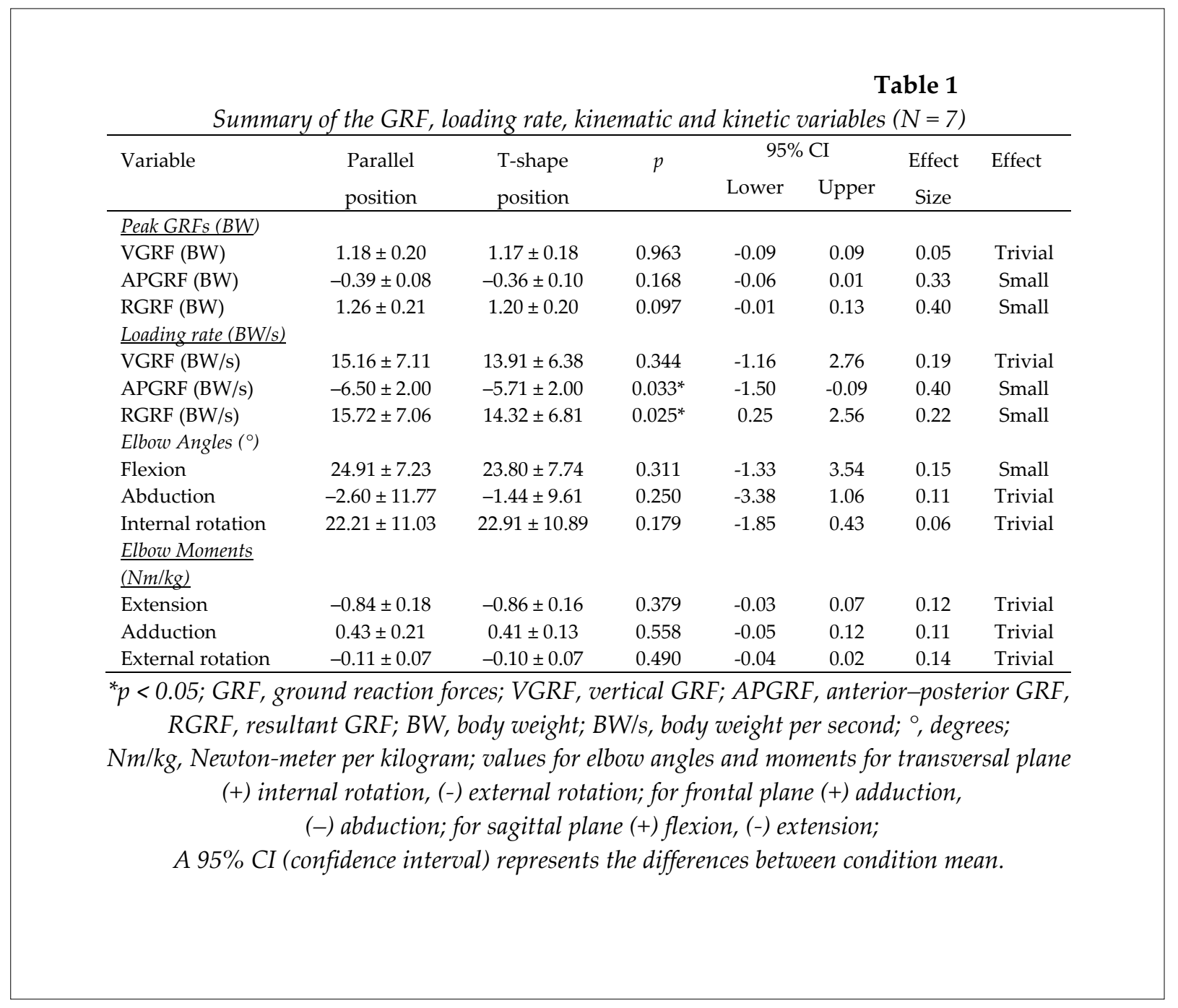

\section{Discussion}

Elbow injury in gymnastics is a potential career ending injury and represents a meaningful risk to the longevity of sports performers. Building on research that has examined the mechanical injury risk of the second contact limb (Farana et al., 2014), the aim of this study was to investigate elbow joint injury risk factors during the first contact limb for both parallel and T-shape $\mathrm{RO}$ techniques.

The hypothesis highlighted that different hand positions of the second contact limb would affect the mechanics of the first contact limb elbow joint, revealing injury risks that were currently not apparent. Table 1 and Figures 3A and $3 \mathrm{~B}$ show that for both techniques there were no significant differences in peak VGRF, APGRF, RGRF, and VGRF loading rates. However, there were significant increases in APGRF and RGRF loading rates in the parallel technique (Table 1 and Figure 3B). Although these higher loading rates during the parallel position may be considered an upper extremity injury risk factor, the magnitude of these values was still lower than this previously reported at the second contact limb (Farana et al., 2014). However, the small effect size for these differences in the current study must be taken into account when interpreting these data. With this in mind the findings of the present study are consistent with those of Seeley and Bressel (2005) who stated that the second contact limb during the RO phase of the Yurchenko vault experienced an increase in peak VGRF and APGRF. Although it must be noted that Seeley and Bressel (2005) did not use independent force plates for each hand and the overall support phase was measured only on one force plate (both hands together). The advantage 
of having two independent force plates, as in the current study, allows a more valid inter limb comparison.

In the current study, no significant differences were observed in the abduction angle (Figure 2C) and corresponding internal adduction moment (Figure 3D) for the first contact limb (Table 1). Moreover, when comparing the first and second contact limb elbow internal adduction moment reported by Farana et al. (2014), there is a decrease by $0.32 \mathrm{~N} / \mathrm{kg}$ in the parallel technique. Within gymnastics research, these factors have been previously associated with elbow joint injury risk factors (Farana et al., 2014; Koh et al., 1992). Interestingly, a large standard deviation in the abduction angle for the first contact limb was observed and indicated high intra and interindividual variability for both techniques (Table 1 and Figure 3C). Although the movement variability is an important factor from an injury prevention perspective (Farana et al., 2015; Hamill et al., 1999), a possible explanation for this might be that the variability observed in the first limb could provide a mechanism to control the variability and load in the second limb. This pattern of variability has been previously observed in other gymnastics skills where end point variability is reduced to control skilled performance (Hiley and Yeadon, 2012).

In comparison to the second limb (Farana et al., 2014), there is a decrease in the first contact limb internal rotation in both techniques. The difference in the transverse plane movement patterns between the first and second limb highlights the greater first contact limb external rotation. Consistency in the first limb external rotation between the two techniques is in contrast to the differences reported with regard to the second limb, i.e. limb abduction loading of the elbow decreases with the T-shape technique (Farana et al., 2014). In terms of the aetiology of elbow injury, a higher abduction load, as reported during the parallel technique, has been associated with the marker of medial collateral ligament strain (Hume et al., 2006; Hurd et al., 2011) predisposing gymnasts to traction injuries on the medial side and compressive injuries to the posterior and lateral structures (Farana et al., 2014).

In both techniques, a higher elbow extension moment was observed for the first contact limb compared to the second contact limb (Farana et al., 2014). It may be speculated that in combination with relatively low elbow flexion in the first contact limb, there may be an increase in loading on the extensor muscles that further may increase the risk of posterior elbow injuries (e.g. triceps tendinitis) (Badia and Stennett, 2006). The reduced elbow flexion in the first contact limb did not expose the elbow joint complex to an increased abduction load, which is in contrast to the role of elbow flexion highlighted by Fornalski et al. (2003).

Findings from the current study show that the first contact limb is exposed to a lower mechanical load and further reinforces and supports the use of the T-shape technique of the RO skill (Farana et al., 2014, 2015). When considered in relation to previous research, the findings of this study concur with the recommendation that the $\mathrm{T}$-shape technique may help reduce injury. These results have implications for coaches and clinicians, when potential risk factors are identified and the process of technique selection is made to be more objective and safe. Conclusions from this study must be considered with the sample size in mind; however, the current study benefited from the use of elite international level gymnasts and has a high degree of ecological validity. In addition, different performance levels, genders and stages of learning need to be considered as factors that may influence the occurrence of injury.

The current study extends knowledge and understanding about different hand positions during the RO skill in female gymnastics. These results demonstrate that technique selection in the RO skill did not influence first contact limb elbow joint mechanics and external forces. These findings suggest that technique selection for the fundamental gymnastics skill of the RO would be better focused on the second hand contact and that the first hand does not act as an influencing factor. The combination of ecological validity and a scientific approach provides a useful insight into technique selection that will help coaches, athletes and clinicians. 


\section{Acknowledgements}

The authors would like to thank Dr. Timothy Exell (Department of Sport and Exercise Science, University of Porthsmounth) and Dr. Gerda Strutzenberger (Department of Sport Science and Kinesiology, Univesity of Salzburg), for their assistance and help in this research. The authors also thank the gymnasts who participated in the study. This research was supported by a Czech Science Foundation (GACR) [project no. 16-14133Y].

\section{References}

Badia A, Stennett C. Sports-related injuries of the elbow. J Hand Ther, 2006; 19(2): 206-226

Chan D, Aldridge MJ, Maffuli N, Davies AM. Chronic stress injuries of the elbow in young gymnasts. $\mathrm{Br} \mathrm{J}$ Radiol, 1991; 64(768): 1113-1118

Cohen J. Statistical power analysis for the behavioural sciences, 2nd ed, Hillsdale: Lawrence Erlbaum; 1988

Cossens P. Injury prevention in artistic gymnastics: A guide for coaches and directions for research. In Bradshaw EJ, Burnett A, \& Hume PA, editors. XXX international symposium of biomechanics in sports. Melbourne, Australia: Australian Catholic University; 2012

Daly RM, Rich PA, Klein R, Bass R. Effects of high-impact exercise on ultrasonic and biochemical indices of skeletal status: A prospective study in young male gymnasts. J Bone Miner Res, 1999; 14(7): 1222-1230

Davidson PL, Mahar B, Chalmers DJ, Wilson BD. Impact modeling of gymnastic back-handsprings and diverolls in children. J Appl Biomech, 2005; 21(2): 115-128

DiFiori JP, Caine DJ, Malina RM. Wrist pain, distal radial physeal injury, and ulnar variance in the young gymnast. Am J Sports Med, 2006; 34(5): 840-849

Farana R, Jandacka D, Uchytil J, Zahradnik D, Irwin G. The influence of hand positions on biomechanical injury risk factors at the wrist joint during the round-off skills in female gymnastics. J Sport Sci, 2016; In press

Farana R, Irwin G, Jandacka D, Uchytil J, Mullineaux D. Elbow joint variability for different hand positions of the round off in gymnastics. Hum Mov Sci, 2015; 39: 88-100

Farana R, Jandacka D, Irwin G. Influence of different hand positions on impact forces and elbow loading during the round off in gymnastics: a case study. Sci Gymnastics J, 2013; 5(2): 5-14

Farana R, Jandacka D, Uchytil J, Zahradnik D, Irwin G. Musculoskeletal loading during the round-off in female gymnastics: The effect of hand position. Sports Biomech, 2014; 13(2): 123-134

Fédération Internationale de Gymnastique (FIG). 2013-2016 Code of points: Women's artistic gymnastics, Lausanne: Switzerland; 2013

Fornalski S, Gupta R, Lee TQ. Anatomy and biomechanics of the elbow joint. Tech Hand Up Extrem Surg, 2003; 7(4): 168-178

Frostick SP, Mohammad M, Ritchie DA. Sport injuries of the elbow. Br J Sports Med, 1999; 33(5): 301-311

Hamill J, van Emmerik RE, Heiderscheit BC, Li L. A dynamical systems approach to lower extremity running injuries. Clin Biomech, 1999; 14(5): 297-308

Hiley M, Yeadon MR. Achieving consistent performance in a complex whole body movement: The Tkatchev on high bar. Hum Mov Sci, 2012; 31: 834-843

Hopkins WG. Measures of Reliability in Sports Medicine and Science. Sports Med, 2000; 30(1): 1-15

Hume PA, Reid D, Edwards T. Epicondylar injury in sport. Sports Med, 2006; 36: 151-170 
Hurd WJ, Kaufman KR, Murthy NS. Relationship between the medial elbow adduction moment during pitching and ulnar collateral ligament appearance during magnetic resonance imaging evaluation. $A m$ J Sports Med, 2011; 39(6): 1233-1237

Irwin G, Exell TA, Manning ML, Kerwin D. Biomechanical evolution of the Tkachev on uneven bars in female gymnastics. International Biomechanics, 2015; 1(1): 21-28

Jackson DW, Silvino N, Reiman P. Osteochondritis in the female gymnast's elbow. Arthroscopy, 1989; 5(2): $129-136$

Koh TJ, Grabiner MD, Weiker GG. Technique and ground reaction forces in the back handspring. Am J Sports Med, 1992; 20(1): 61-66

Lindner KJ, Caine DJ. Injury patterns of female competitive club gymnasts. Can J Sport Sci, 1990, 15(4): 254261

Magra M, Caine D, Maffulli N. A review of epidemiology of paediatric elbow injuries in sports. Sports Med, 2007; 37(8): 717-735

Panzer VP, Bates BT, McGinnis PM. A biomechanical analysis of elbow joint forces and technique differences in the Tsukahara vault. In: Hoshizaki TB, Salmela JH, Petiot B, editors. Diagnostics, treatment and analysis of gymnastic talent. Montreal, Canada: Sport Psyche Editions; 1987

Sands WA, McNeal JR. Hand position in a back handspring. Technique, 2006; 26(3): 8-9

Seeley MK, Bressel E. A comparison of upper-extremity reaction forces between the Yurchenko vault and floor exercise. J Sports Sci Med, 2005; 4(2): 85-94

Selbie SW, Hamill J, Kepple TM. Research Methods in Biomechanics. Champaign, IL: Human Kinetics, Chapter 7; 151-176; 2014

Webb BG, Rettig LA. Gymnastic wrist injuries. Curr Sports Med Rep, 2008; 7(5): 289-295

\section{Corresponding author:}

\section{Dr. Roman Farana}

Department of Human Movement Studies, Human Motion Diagnostic Center, University of Ostrava, Czech Republic, 70103 Moravska Ostrava,

Tel: +420 59709 2593,

E-mail: Roman.Farana@osu.cz) 